\title{
Maternal administration of cannabidiol promotes an anti-inflammatory effect on the intestinal wall in a gastroschisis rat model
}

\author{
G.H. Callejas ${ }^{1}$, R.L. Figueira ${ }^{1}$, F.L.L. Gonçalves ${ }^{1}$, F.A.P. Volpe ${ }^{1}$, A.W. Zuardi ${ }^{2}$, J.A. Crippa $^{2}$, \\ J.E. Hallak ${ }^{2}$ and L. Sbragia ${ }^{1}$ \\ ${ }^{1}$ Laboratório de Cirurgia Experimental Fetal e Neonatal "Michael Harrison” Divisão de Cirurgia Pediátrica, Departamento de \\ Cirurgia e Anatomia Faculdade de Medicina de Ribeirão Preto, Universidade de São Paulo, Ribeirão Preto, SP, Brasil \\ ${ }^{2}$ Departmento de Neurociências e Comportamento, Faculdade de Medicina de Ribeirão Preto, Universidade de São Paulo, \\ Ribeirão Preto, SP, Brasil
}

\begin{abstract}
Gastroschisis (GS) is an abdominal wall defect that results in histological and morphological changes leading to intestinal motility perturbation and impaired absorption of nutrients. Due to its anti-inflammatory, antioxidant, and neuroprotective effects, cannabidiol (CBD) has been used as a therapeutic agent in many diseases. Our aim was to test the effect of maternal CBD in the intestine of an experimental model of GS. Pregnant rats were treated over 3 days with CBD $(30 \mathrm{mg} / \mathrm{kg})$ after the surgical induction of GS (day 18.5 of gestation) and compared to controls. Fetuses were divided into 4 groups: 1) control (C); 2) C + CBD (CCBD); 3) gastroschisis (G), and 4) G + CBD (GCBD). On day 21.5 of gestation, the fetuses were harvested and evaluated for: a) body weight (BW), intestinal weight (IW), and IW/BW ratio; b) histometric analysis of the intestinal wall; c) immunohistochemically analysis of inflammation (iNOS) and nitrite/nitrate level. BW: GCBD was lower than CCBD (P<0.005), IW and IW/ BW ratio: GCBD was smaller than $G(P<0.005)$, GCBD presented lower thickness in all parameters compared to $G(P<0.005)$, iNOS and nitrite/nitrate were lower concentration in GCBD than to $G(P<0.005)$. Maternal use of CBD had a beneficial effect on the intestinal loops of GS with decreased nitrite/nitrate and iNOS expression.
\end{abstract}

Key words: Gastroschisis; Experimental model; Prenatal treatment; Cannabidiol

\section{Introduction}

Gastroschisis (GS) is a congenital abdominal wall defect characterized by a small hole, generally located to the right of the navel, resulting in the herniation and permanent exposure of the bowel loops to amniotic fluid and its components during pregnancy (1). This exposure promotes morphological and histological changes to the intestinal wall, such as reduction of intestine length, thickening of intestinal layers, and disturbance of neuronal cells maturation, leading to intestinal hypomotility and impaired absorption of nutrients, which in turn raises the occurrence of post-operatory complications, elevating the morbidity, mortality, and time of stay treatment cost in the neonatal period $(2,3)$.

Simple GS may have a survival rate close to $100 \%$. On the other hand, association with malrotation, infarction, atresia, perforation, and stenosis has a mortality rate of $28 \%$, besides higher risk of long-term complications such as short gut syndrome (1). Molecular mechanisms involved in GS have not been completely unraveled.
However, it is known that its inflammatory process causes histological and physiological changes associated with high local concentration of nitric oxide (NO) and nitric oxide synthases (NOS) (4).

$\mathrm{NO}$ is a free radical gas that has numerous molecular targets and a short half-life in biological systems. As a method to indirectly measure the levels of NO, the concentration of nitrite, a stable product of NO formation, in blood and tissue has been used as routine $(5,6)$.

In normal conditions, NO and NOS are expressed at low levels. NO promotes smooth muscle relaxation and it is involved with epithelial permeability, whereas at high levels NO can affect the gut barrier permeability leading to increased bacterial translocation and epithelial damage. NO production is mediated mainly by two NOS, eNOS (endothelial) and iNOS (induced) through catalysis. eNOS is constitutive, while iNOS is upregulated during inflammatory processes increasing NO production (6-8). 
Prevention or reduction of the inflammatory intestinal fetal lesion has been a recent target of treatment strategy in experimental GS. Maternal administration of corticosteroids showed, beside the anti-inflammatory action, favorable effects upon fetal pulmonary and intestinal development $(2,3,9,10)$. However, this strategy may lead to deleterious effects such as increased maternal infection (11), brain growth deficits, periventricular leukomalacia, poor attention and cognitive performance (12), and low neuropsychiatric functioning (13). Thus, there is a clear need to explore new pharmacological possibilities for antenatal management.

Cannabidiol (CBD) possesses anti-inflammatory, antioxidant and neuroprotective effects with a wide range of possible therapeutic effects in diseases such as Parkinson's, Alzheimer's, brain ischemia, diabetes, nausea, cancer, and rheumatoid arthritis (14). This cannabinoid has shown a favorable safety profile in humans (15). CBD can modulate tumor necrosis factor in vitro and suppress the production of chemokines by human B cells in a model of rheumatoid arthritis in synovial cells isolated from treated knees (16). In acute inflammation and experimental chronic pain, it has had a beneficial effect upon edema and hyperalgesia $(17,18)$. Considering the bowel loops inflammation and the immaturity of the myenteric plexus that occur in fetal GS $(19,20)$, our aim was to evaluate the effect of maternal administration of $\mathrm{CBD}$ in an experimental rat model of the disease.

\section{Material and Methods}

This study was approved by the Ethics Committee in Animal Experimentation of Faculdade de Medicina de Ribeirão Preto, Universidade de São Paulo, Ribeirão Preto, SP, Brazil (\#215/2014).

\section{Animals}

Female Sprague-Dawley rats were submitted to mating. A male/female pair was maintained together overnight. The following morning, a vaginal swab was performed and the presence of sperm marked day zero of pregnancy (time of gestation, 22 days).

\section{Groups}

A total of 10 pregnant rats were operated, resulting in 40 fetuses that were subdivided in 4 groups $(n=10$ per group): 1) control (C); 2) control + CDB (CCBD); 3) gastroschisis (G), and 4) gastroschisis + CBD (GCBD). The maternal administration of CBD was $30 \mathrm{mg} / \mathrm{kg}$ via intraperitoneal (ip) injection, once per day for 3 days (from surgical day 18.5 until harvest day 21.5 ).

\section{Gastroschisis surgical procedure}

Surgery was performed at 18.5 days of gestation. Pregnant rats were anesthetized with $50 \mathrm{mg} / \mathrm{mL}$ ketamine (Ketamina $^{\circledR}$, Pfizer, Brazil) and $10 \mathrm{mg} / \mathrm{mL}$ xylazine (Rompum $^{\mathbb{R}}$, Bayer, Brazil). The abdominal cavity was opened by median laparotomy in 2 layers. Fetuses were counted from the uterine isthmus, and GS was created on the second fetus of each horn according to the technique described by Correia-Pinto et al. (21). The lower body of the fetus was exposed and a right para-umbilical laparotomy with standardized extension of $5 \mathrm{~mm}$ was performed, opening the fetal abdominal cavity with caution not to damage the umbilical vessels and liver. The bowel loops were gently exposed by the delicate compression of the fetal abdomen with cotton swabs. After creating the defect, the fetus was carefully placed back into the uterine cavity, and a previously placed purse string suture closed the uterus.

\section{CBD Preparation}

CBD was provided in powder form with $99.9 \%$ purity (BSPG-Pharm, UK). The drug was suspended in polyoxyethylenesorbitan monooleate (Tween 80 ) $2 \%$ saline, at a concentration of $30 \mathrm{mg} / \mathrm{kg}$. The concentration was based on previous studies in rat neonates and adapted to this model (22). CBD was administrated in the pregnant rat by ip injection on $18.5,19.5$, and 20.5 days of gestation.

\section{Harvest}

Pregnant rats were submitted to harvesting on day 21.5. Fetuses were removed, weighed, and dissected to harvest the ileum. The intestinal segment was removed from ileum until the proximal colon, approximately $2 \mathrm{~cm}$. The tissue was then fixed in $10 \%$ formalin solution for immunohistochemistry and histologic analysis, and frozen for nitrite/nitrate analysis.

\section{Morphological and histometric analysis}

Body weight (BW), intestinal weight (IW), and the IW/ BW ratio were analyzed $(n=10)$. For weight analysis, the whole intestine was considered (wet weight). The intestinal histology samples from ileum were stained by Masson's trichrome and photographed using the Nikon Eclipse E200 80 i photomicroscope (Nikon, Japan), with a $400 \times$ magnification for measurement of the intestinal wall layers. The images were digitized, allowing measurement of the layers serosa (SE), longitudinal muscle (LM), circular muscle (CM), submucosal mucous membrane (SM), and total wall (TL) using Image Pro Plus 6.0 software (Media Cybernetics Inc., USA). Twelve fields per slide were analyzed in a total of 4 slides per group.

\section{iNOS Immunohistochemical analysis}

Twelve fields per slide and four slides per group were analyzed by three blind investigators. The immunohistochemistry score was graded according to the intensity of staining from 0 to 4 : $0=$ =negative, $1=$ weak staining, $2=$ moderate staining, $3=$ strong staining, 4=very strong staining (23).

The slides were deparaffinized in xylol and dehydrated with ethanol. After these steps, blocking of endogen peroxidases were prepared by incubation of the slides in $\mathrm{H}_{2} \mathrm{O}_{2}(30 \%)$ and $3 \%$ methanol for $10 \mathrm{~min}$. For antigenic exposure, the slides were placed in $50 \mathrm{mM}$ Tris- $\mathrm{HCl}$ buffer 
solution, pH 9.5 (containing 5\% urea) and in an Optisteam Plus steamer, model 652, (Krups North America, USA) for $40 \mathrm{~min}$, then cooled in an ice water bath for $15 \mathrm{~min}$, and lastly washed in distilled water. Blocking was performed with $10 \%$ goat serum in phosphate buffered saline (PBS, $\mathrm{Na}_{2} \mathrm{HPO}_{4} 20 \mathrm{mM}+\mathrm{NaCl} 0.45 \mathrm{M}, \mathrm{pH} 7.4$ ) for $30 \mathrm{~min}$ in a humidity chamber. The slides were incubated overnight at $4^{\circ} \mathrm{C}$ with the primary antibody (rabbit anti-iNOS sc-651 Santa Cruz Biotechnology, USA) diluted 1:200 in PBS with $3 \%$ bovine serum albumin (BSA). After removal of the primary antibody with PBST $2 \%$ solution, a Polymer Detection Kit (MACH 4 Universal HRP-Polymer M4U534, Biocare Medical, USA) was added for $30 \mathrm{~min}$. The printing was performed with a solution of $3,3^{\prime}$-diaminobenzidinetetra-hidrocloret (Sigma, USA) and $0.03 \%$ hydrogen peroxide in triphosphate buffered saline (TBS) $0.05 \mathrm{M}, \mathrm{pH} 7.6$, for $5 \mathrm{~min}$. Lastly, the slides were counter-stained with Harris hematoxylin, washed in running water, dehydrated by a series of ascending concentrations of alcohol and xylol baths, and cover slipped with Permount ${ }^{\mathbb{R}}$ (Fisher Scientific, USA).

\section{Sample preparation for Griess reaction}

Each $100 \mu \mathrm{g}$ of intestine was homogenized in $400 \mu \mathrm{L}$ of buffer containing $10 \mathrm{mM}$ EDTA, $100 \mathrm{mM}$ Trizma base, $10 \mathrm{mM}$ sodium pyrophosphate, $100 \mathrm{mM}$ sodium fluoride, $10 \mathrm{mM}$ sodium orthovanadate, $0.1 \mathrm{mg} / \mathrm{mL}$ aprotinin, $2 \mathrm{mM}$ phenylmethylsulfonyl fluoride, and distilled water then centrifuged in a Mikro 200R centrifuge (Hettich, Germany) at $12,000 \mathrm{rpm}$ at $4^{\circ} \mathrm{C}$ for $30 \mathrm{~min}$; the supernatant was aliquoted for analysis. To avoid the process of tyrosine nitration and S-nitrosocysteine formation observed in biological samples submitted to slow freezing, we rapidly froze our samples in liquid nitrogen (11).

\section{Determination of nitrite/nitrate concentration by Griess reaction}

The concentration of nitrite/nitrate $\left(\mathrm{NO}_{2} / \mathrm{NO}_{3}\right)$ was performed from the terminal ileum homogenate by the Griess reaction after enzymatic reduction of nitrate to nitrite by nitrate reductase enzyme (Sigma, USA) in a reducing solution containing $0.5 \mathrm{M}$ phosphate buffer, $\mathrm{pH} 7.5$, and NADPH. For this reaction, a 96-well flat bottom (USA Corning, USA) plate was used in which were deposited $40 \mu \mathrm{L}$ of the reducing solution and $40 \mu \mathrm{L}$ of each sample in triplicate, and maintained at $37^{\circ} \mathrm{C}$ for $12 \mathrm{~h}$. Then, nitrite was detected through the addition of $20 \mu \mathrm{L}$ of Griess reagent. After $10 \mathrm{~min}$, the absorbance was measured at $540 \mathrm{~nm}$ using an automatic microplate reader (Molecular Devices, Spectra Max 250, USA). Nitrite concentrations were calculated based on the standard curve of $\mathrm{NaNO}_{2}$ and data are reported as $\mu \mathrm{M} / \mu \mathrm{g}$ intestinal tissue (24).

\section{Statistical analysis}

For morphological, histological, and nitrite/nitrate data analysis, ANOVA and Tukey's post-test were used. For immunohistochemistry concentration data, the Kruskal
Wallis with Dunn's post-test were used. Significance was considered when $P<0.05$. The software used was GraphPad Prism 6.0 (GraphPad Software Inc., USA). Results are reported as means $\pm S D$, and median and interquartile range (IQR) for immunohistochemistry analysis.

\section{Results}

\section{Morphological and histometric analysis}

Figure 1 shows macroscopical and microscopical differences of the bowel loop between $\mathrm{G}$ and GCBD groups.

A significant difference was found for BW between CCBD vs GCBD (4.142 \pm 0.598 vs $3.270 \pm 0.683$ g, respectively $\mathrm{P}<0.005$ ). For IW analysis, the GCBD group showed reduced values $(0.124 \pm 0.037 \mathrm{~g})$ compared to the $\mathrm{G}$ group $(0.190 \pm 0.033 \mathrm{~g}, \mathrm{P}<0.005)$ and similar to the other groups $C$ and CCBD $(0.107 \pm 0.008$ and $0.126 \pm 0.021 \mathrm{~g}$, respectively, $\mathrm{P}>0.05)$. The GCBD group showed lower IW/BW ratio $(0.038 \pm 0.008)$ compared to $G$ group $(0.053 \pm 0.010$, $\mathrm{P}<0.005)$ and higher than the $C$ group $(0.029 \pm 0.002)$, and there was no difference to the CCBD group (0.030 \pm 0.004 , $P>0.05)$. Results are shown in Figure 2.

In the histometric analysis of tissue layers, (SE, ML, $\mathrm{CM}, \mathrm{SM}$, and TL), the GCBD group presented reduced thickness compared to group $\mathrm{G}$, and no difference compared to groups $\mathrm{C}$ and $\mathrm{CBD}$ in any of the layers (Figure 3 ).

\section{iNOS immunohistochemical analysis}

The group GCBD showed reduced iNOS activity in comparison to the $\mathrm{G}$ group, and similar to the groups $\mathrm{C}$ and CCBD (Figure 4).

\section{Nitrite/nitrate concentration of the intestine tissue homogenate}

The GCBD group showed reduction of $\mathrm{NO}_{2} / \mathrm{NO}_{3}$ concentration compared to group $\mathrm{G}$ and was similar to groups $\mathrm{C}$ and CCCB, (56.862 \pm 14.644 vs $122.377 \pm 15.463$, $\mathrm{P}<0.005,55.249 \pm 29.607$ and $53.120 \pm 15.089, \mathrm{P}>0.05$, respectively, Figure 5).

\section{Discussion}

GS is an abdominal wall defect that occurs in $1 / 4,000$ live births and is increasing all over the world $(25,26)$. Maternal therapy with dexamethasone in a GS rat model showed reversion of intrauterine growth restriction (IUGR), reduction of intestinal weight and mucosal thickness $(3,5)$. Treatment with hydrogel associated to an NO donor covering the GS fetal bowel loops as well as low concentration release of $\mathrm{NO}$ in the amniotic fluid reduced morphological parameters and intestinal wall thickness $(4,27)$.

Enteric glial cells actively mediate acute and chronic inflammation in the intestine promoting proliferation and release of neurotrophins, growth factors and pro-inflammatory chemokines that in turn may increase the immunological 

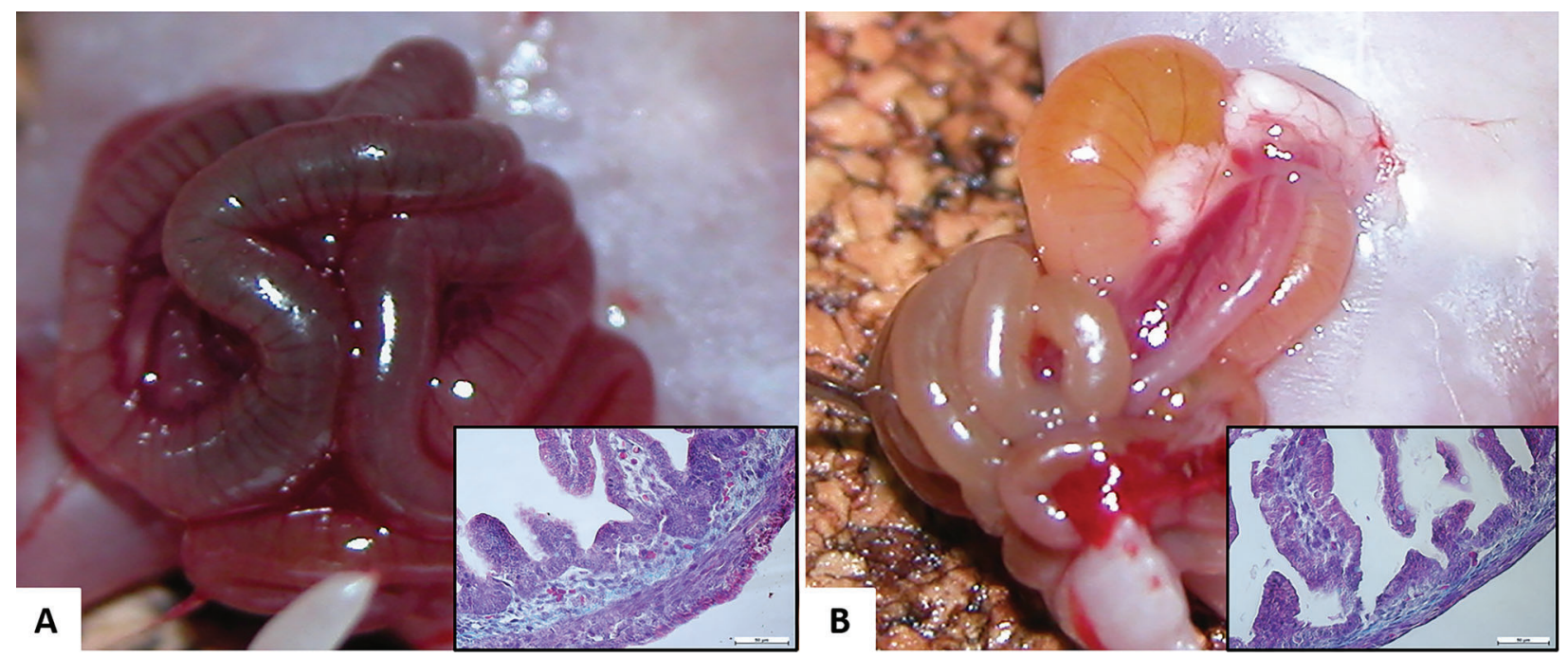

Figure 1. $A$, Macroscopic view of the intestinal evisceration of the gastroschisis (G) group (time of harvest). Inset: histological representation of ileum specimen stained with $H \& E$ of the $G$ group. $B$, Macroscopic view of the intestinal evisceration of the gastroschisis + cannabidiol (GCBD) group (time of harvest). Inset: histological representation of the ileum specimen stained with H\&E of the GCBD group. Note the different aspect and thickness of the bowel loop macroscopically and microscopically between the 2 groups. Masson's trichrome staining from ileum segment. Magnification: $100 \times$. Scale bar: $10 \mu \mathrm{m}$.
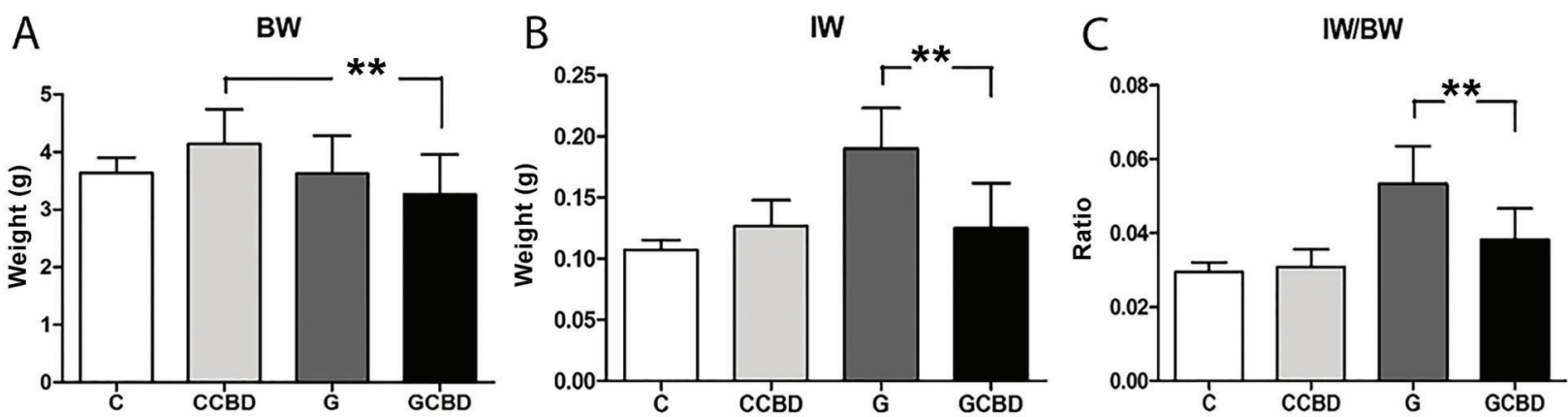

Figure 2. Morphological analysis. Data are reported as means $\pm \mathrm{SD}$. $A$, body weight (BW; g). $B$, intestinal weight (IW, g). $C$, Intestinal weight/body weight ratio (IW/BW). C: control; CCBD: control+cannabidiol; G: gastroschisis; GCBD: gastroschisis + cannabidiol. ${ }^{* *} \mathrm{P}<0.005$ (ANOVA).

response, representing a very important link between the nervous and immune system in the intestine. CBD is an interesting compound due to its capacity to control reactive gliosis in the central nervous system, without psychotropic effects and safe toxicological profile $(15,28,29)$.

In this study, we verified that maternal intraperitoneal administration of CBD had no effect on BW, which showed difference only between the group CCBD and GCBD, with the former presenting higher values. While the effect of CBD in IUGR in clinical and experimental fetuses is not well known, it has been demonstrated that longer exposure to CBF of the amniotic fluid worsens IUGR in an experimental model in rats (30), which might be linked to fetal alterations responsible for glucose and insulin receptors modification by fetal inflammation (31). Such modification could mean that CBD injection, even if intraperitoneal, in a control (healthy) organism in growth phase could have an effect on body mass gain, without the same effect seen in the GCBD group (treated disease). Wasserman et al. (32) verified, in C57bl/6j mice with ages between 5 and 11 weeks, that cannabinoid receptors CB1 and CB2 are specifically located in hypertrophic chondrocytes of the epiphyseal growth cartilage, which drives vertebrate growth. Additionally, blocking CB2 receptors slows skeleton elongation, which could explain body growth changes in the healthy newborns treated with CBD found in our research. It is important to highlight that the increase of BW in the CCBD group was only significant when compared to the GCBD group, and that, when compared to the $C$ group, did not show differences in any of the parameters (BW, IW, IW/BW). 
a

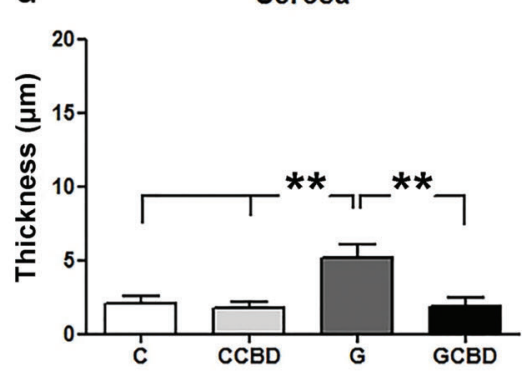

b Longitudinal muscle layer

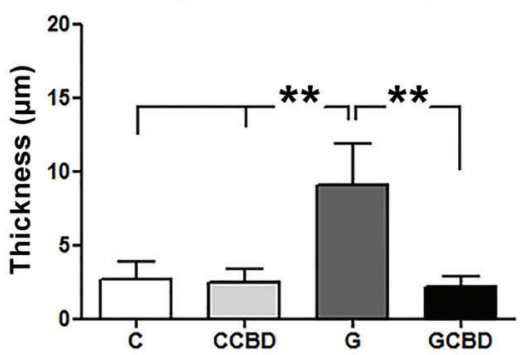

C

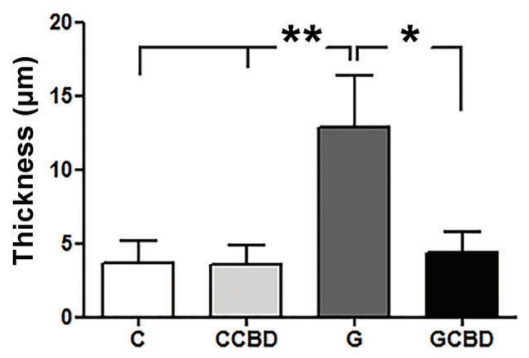

d

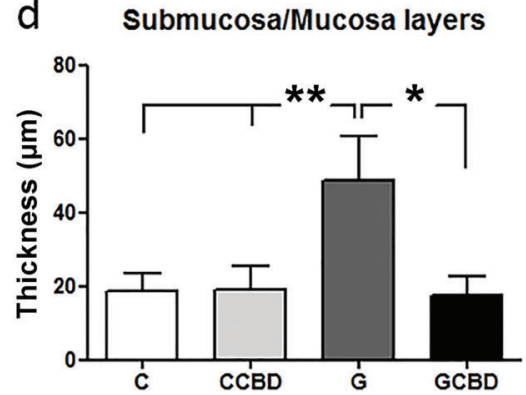

e

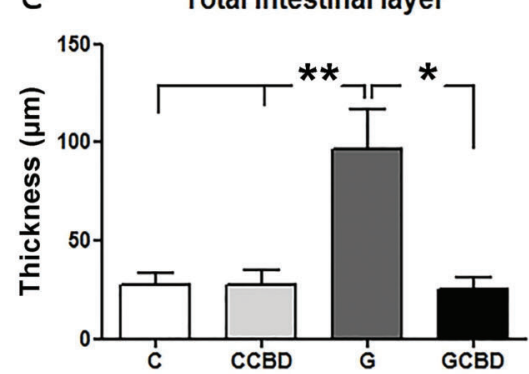

Figure 3. Histometric analysis of the intestinal layers. Data are reported as means \pm SD. C: Control; CCBD: Control + cannabidiol; G: Gastroschisis; GCBD: Gastroschisis + cannabidiol. ${ }^{*} \mathrm{P}<0.05 ;{ }^{* *} \mathrm{P}<0.005$ (ANOVA).

At first, this could be an evidence that this treatment has no risk of impairment related to weight.

An increased IW and IW/BW ratio was observed in the G group compared to the other groups (C, CICBD, GCBD) and a reduction was observed in the GCBD group compared to $G$. Such findings might demonstrate that CBD use resulted in benefits to the GCBD group with a potential reversion of the inflammatory reaction caused by the exposure of the bowels to the amniotic fluid. Additionally, no significant difference was found between groups GCBD and $C$ in any of the parameters. Therefore, the results of the morphological analysis demonstrated potential benefits induced by the treatment with CBD.

Furthermore, the histometric analysis showed a reduction of the intestinal layer thickness in the GCBD group compared to the $G$ group and had similar results to the other control groups. The normality of this parameter of intestinal inflammation corroborates the hypothesis that CBD not only was effective in reducing the intestinal inflammation, but also did not cause any impairment to intestinal development.

The iNOS expression was reduced in group GCBD in relation to $\mathrm{G}$ group. The increased NOS expression in GS could mediate hypoperistalsis and malabsorption (33). In addition, a study showed that inhibition of NOS was correlated with reduction of intestinal thickness and improvement of bowel loops macroscopic appearance of GS in chick embryos (34). In previous studies, we had observed the reduction of iNOS in GS fetuses after treatment with a NO donor (20). In the literature, the inhibiting effect of CBD over iNOS has previously been described in an in vivo model of neuroinflammation; mice that suffered beta-amyloid protein-induced neurological inflammation received treatment with intraperitoneal CBD and showed reduction of iNOS expression in relation to the non-treated diseased group (35).

However, a study that evaluated the effect of CBD in a neuropathic pain model and sciatic nerve lesion did not show reduction of iNOS expression with $\mathrm{CBD}$, suggesting that the relationship between CBD and NOS might show peculiarities in different tissues. Rats with inflamed paws or sciatic nerve lesion had an increase of iNOS, nNOS, and eNOS expression. Treatment with CBD only reduced the overexpression of eNOS in rats with inflamed paws, without reaching similar levels as the control group, while there was no significant reduction of iNOS and nNOS (17).

A diabetes neuropathy study in rats suggests that NOS could have an inhibitory effect over cannabinoid receptor agonists. According to the authors, the use of a specific nNOS inhibitor (7-Ni) and a relatively selective inhibitor of iNOS (L-NIL) showed a positive impact over the analgesic effect of two cannabinoid receptors agonists. Such finding suggests that CBD might not only modulate the expression and activity of NOS isoforms, but also have an inhibitory effect on the analgesia and anti-inflammatory pathways activated by cannabinoids agonists (36). In this study, we observed the reduction of nitrite/nitrate concentration in the GCBD group in comparison to the $G$ group; values were similar to the groups $C$ and $C B D$, which indirectly shows the reduction of $\mathrm{NO}$ concentration in the treated group in 

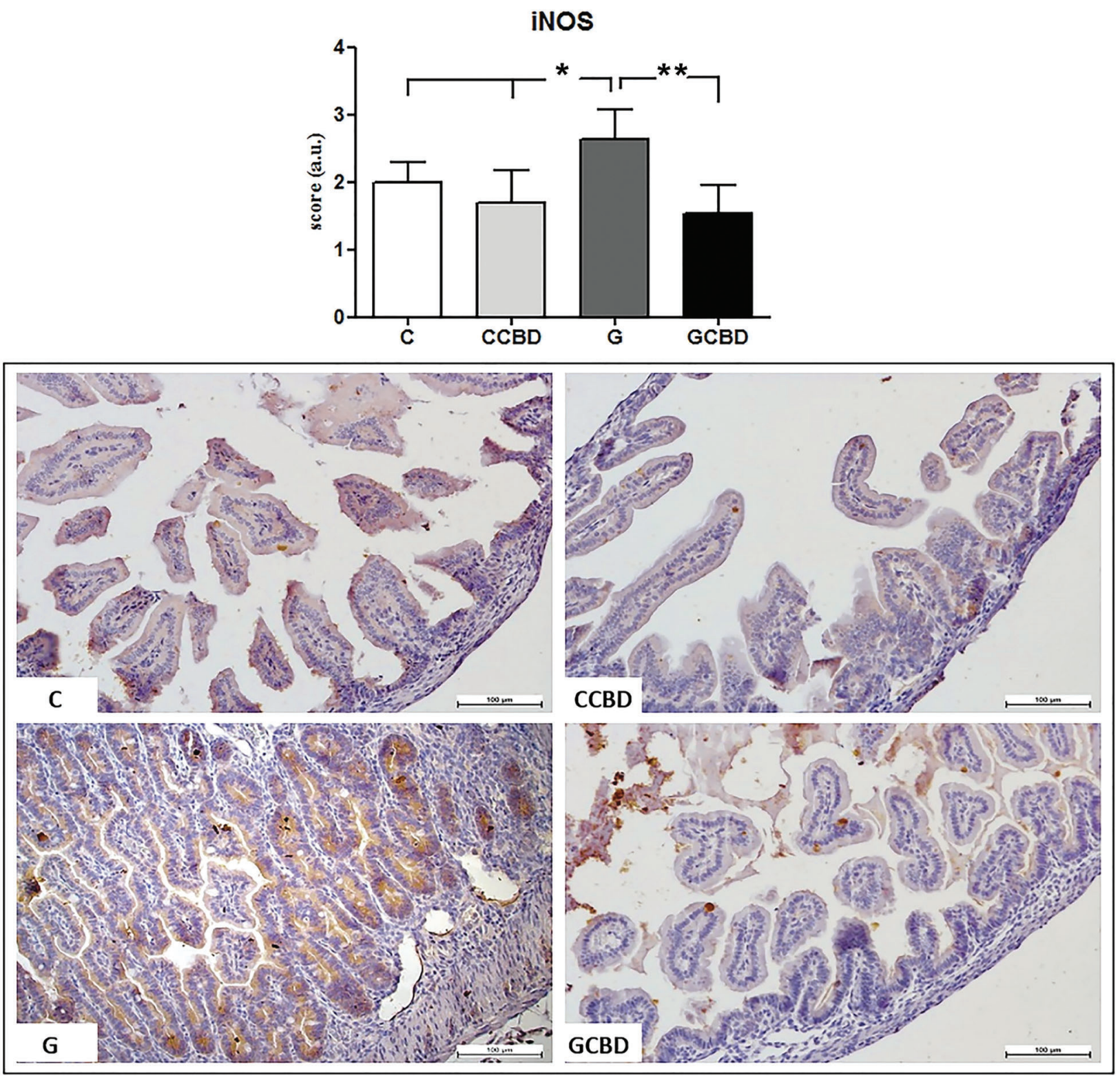

Figure 4. Immunohistochemistry score for iNOS expression and the histological transversal slides stained by immunohistochemistry. Data are reported as means \pm SD. Magnification $200 \times$, bar $=100 \mu \mathrm{m}$. C: control; CCBD: control + cannabidiol; G: gastroschisis; GCBD: gastroschisis + cannabidiol. ${ }^{*} \mathrm{P}<0.05 ;{ }^{* *} \mathrm{P}<0.005$ (Kruskal Wallis).

comparison to the diseased non-treated group. The NO reduction could be explained by the decrease of cytotoxic action mediated by macrophages and other immune cells, but further studies should be made to confirm this hypothesis.

CBD does not modify intestinal motility in control animals, but can inhibit the hypermotility associated to experimental ileitis in mice (37). Borrelli et al. (38) evaluated the CDB effect in experimental colitis in mice and verified a reduction of intestinal inflammation shown by the reduction of iNOS and the interleukins 1-beta and 10, as well as reduction of the intestinal mucous oxidative stress. Lin et al. evaluated the activity of the cannabinoid receptor G Protein-coupled Receptor 55 (GPR55) and its ligands O-1602 in the gut movement of rodents with lipopolysaccharide (LPS)-induced intestinal inflammatory disease and concluded that CBD selectively normalizes motility perturbation through potential mechanisms involving systemic anti-inflammatory effect and regulation of the myoelectrical activity in the intestine (39). De Filippis et al. (28) also confirmed in human biopsy samples and in mice with 


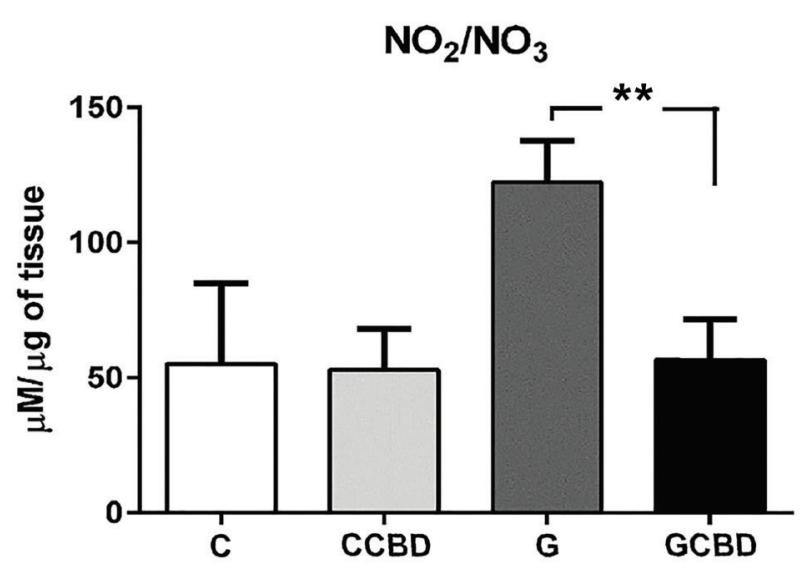

Figure 5. Nitrite/nitrate concentration in intestine tissue homogenate. Data are reported as means \pm SD C: control; CCBD: control + cannabidiol; G: gastroschisis; GCBD: gastrosquisis + cannabidiol. ${ }^{* *} \mathrm{P}<0.005$ (Kruskal Wallis).

LPS-induced enteritis that CBD treatment resulted in reduction of iNOS and S100B.

We aimed to evaluate the CBD impact on intestinal inflammation promoted by amniotic fluid exposure. Our study had some limitations. Although the analyzed parameters were not direct indications of an inflammatory state, they have been previously studied and correlated with GS as a consequence of the inflammation and the cannabidiol treatment showed similar results in comparison to those observed in previous studies that aimed reduction of the inflammation of the intestine $(3,9,10)$. Also, we focused on the intestine responses and thus did not discuss the cannabidiol effects in the brain or other organs, although we can assert that clinical and visible alterations were not observed in the rats.

There was no evidence in the analyzed parameters that the administration of CBD $30 \mathrm{mg} / \mathrm{kg}$ via ip in female pregnant rats have caused impairments to fetal development in the diseased (GCBD) or unaffected groups (CCBD). Although CBD treatment did not correlate with any changes between $C$ and CCBD groups in the evaluated parameters, long term studies are necessary to demonstrate possible secondary effects of CBD.

Finally, the treatment with CDB of experimental GS in rats reduced the weight, thickness of intestinal layers, concentration of $\mathrm{NO}_{2} / \mathrm{NO}_{3}$, and expression of iNOS of the bowel loops showing an effective anti-inflammatory action

\section{References}

1. Dalton BG, Gonzalez KW, Reddy SR, Hendrickson RJ, Iqbal CW. Improved outcomes for in born babies with uncomplicated gastroschisis. J Pediatr Surg 2017; 52: 1132-1134, doi: 10.1016/j.jpedsurg.2016.12.003. and pharmacological application for pre-natal use. Further studies could clarify the interaction between NO and CBD pathways. In addition, investments for future studies translating the present findings to the clinical practice are desirable and suitable.

\section{Declaration of potential conflict of interest}

A.W. Zuardi, J.E. Hallak, and J.A. Crippa are co-inventors (Mechoulam R, JAC, Guimaraes FS, AWZ, $\mathrm{JEH}$, Breuer A) of the patent "Fluorinated CBD compounds", compositions and uses thereof. Pub. No.: WO/2014/108899. International Application No.: PCT/IL2014/050023" Def. US no. Reg. 62193296; 29/07/2015; INPI on 19/08/2015 (BR1120150164927). The University of São Paulo has licensed the patent to Phytecs Pharm (USP Resolution No. 15.1.130002.1.1). The University of São Paulo has an agreement with Prati-Donaduzzi (Toledo, Brazil) to "develop a pharmaceutical product containing synthetic cannabidiol and prove its safety and therapeutic efficacy in the treatment of epilepsy, schizophrenia, Parkinson's disease, and anxiety disorders". JAC and JEH have received travel support from and are medical advisors of BSPG-Pharm.

\section{Acknowledgments}

A.W. Zuardi, J.E. Hallak, J.A. Crippa, and L. Sbragia are recipients of Conselho Nacional de Desenvolvimento Científico e Tecnológico (CNPq) productivity fellowships. The research was supported in part by grants from Fundação de Amparo à Pesquisa do Estado de São Paulo (FAPESP, Processo 2012/09601-4), Conselho Nacional de Desenvolvimento Científico e Tecnológico (CNPq); Coordenação de Aperfeiçoamento de Pessoal de Nível Superior (CAPES); Fundação de Apoio ao Ensino, Pesquisa e Assistência do Hospital das Clínicas da Faculdade de Medicina de Ribeirão Preto da Universidade de São Paulo (FAEPA); Center for Interdisciplinary Research on Applied Neurosciences (NAPNA), University of São Paulo, São Paulo, SP, Brazil; and National Institute for Translational Medicine (INCT-TM; CNPq, Brazil). The present study was supported by a CNPq grant (CNPq/MS/SCTIE/ DECIT No. 26/2014, Pesquisas sobre Distúrbios Neuropsiquiátricos; 466805/2014-4). J.A. Crippa is the recipient of a grant from University Global Partnership Network (UGPN) - Global priorities in cannabinoid research excellence. BSPG-Pharm (Sandwich, UK) has kindly supplied CBD at no cost.

2. Jobe $A H$, Soll RF. Choice and dose of corticosteroid for antenatal treatments. Am J Obst and Gynecol 2004; 190: 878-881, doi: 10.1016/j.ajog.2004.01.044.

3. Yu J, Gonzalez-Reyes S, Diez-Pardo JA. Effects of prenatal dexamethasone on the intestine of rats with gastroschisis. 
J Pediatr Surg 2003; 38: 1032-1035, doi: 10.1016/S00223468(03)00185-4.

4. Gonçalves FL, Bueno MP, Schmidt AF, Figueira RL, Sbragia L. Treatment of bowel in experimental gastroschisis with a nitric oxide donor. Am J Obstet Gynecol 2015; 212: 383. e1-e7, doi: 10.1016/j.ajog.2014.09.025.

5. Bryan NS, Grisham MB. Methods to detect nitric oxide and its metabolites in biological samples. Free Radic Biol Med 2007; 43: 645-657, doi: 10.1016/j.freeradbiomed. 2007.04.026.

6. Hrabák A, Bajor T, Temesi A, Mészáros G. The inhibitory effect of nitrite, a stable product of nitric oxide (NO) formation, on arginase. FEBS Lett 1996; 390: 203-206, doi: 10.1016/ 0014-5793(96)00659-X.

7. Bealer JF, Graf J, Bruch SW, Adzick NS, Harrison MR. Gastroschisis increases small bowel nitric oxide synthase activity. J Pediatr Surg 1996; 31: 1043-1045 ; discussion 1045-1046, doi: 10.1016/S0022-3468(96)90083-4.

8. Grishin A, Bowling J, Bell B, Wang J, Ford HR. Roles of nitric oxide and intestinal microbiota in the pathogenesis of necrotizing enterocolitis. J Pediatr Surg 2016; 51: 13-17, doi: 10.1016/j.jpedsurg.2015.10.006.

9. Yu J, Gonzalez-Reyes S, Diez-Pardo JA. Local dexamethasone improves the intestinal lesions of gastroschisis in chick embryos. Pediatr Surg Int 2004; 19: 780-784, doi: 10.1007/ s00383-003-0958-9.

10. Bittencourt DG, Barreto MW, França WM, Gonçalves A, Pereira LA, Sbragia L. Impact of corticosteroid on intestinal injury in a gastroschisis rat model: morphometric analysis. J Pediatr Surg 2006; 41: 547-553, doi: 10.1016/j.jpedsurg. 2005.11.050.

11. Althabe F, Belizán JM, McClure EM, Hemingway-Foday J, Berrueta M, Mazzoni A et al. A population-based, multifaceted strategy to implement antenatal corticosteroid treatment versus standard care for the reduction of neonatal mortality due to preterm birth in low-income and middleincome countries: the ACT cluster-randomised trial. Lancet 2015; 385: 629-639, doi: 10.1016/S0140-6736(14)61651-2.

12. Damsted SK, Born AP, Paulson OB, Uldall P. Exogenous glucocorticoids and adverse cerebral effects in children. Eur J Paediatr Neurol 2011; 15: 465-477, doi: 10.1016/j.ejpn. 2011.05.002.

13. Drozdowicz LB, Bostwick JM. Psychiatric adverse effects of pediatric corticosteroid use. Mayo Clin Proc 2014; 89: 817-834, doi: 10.1016/j.mayocp.2014.01.010.

14. Zuardi AW. Cannabidiol: from an inactive cannabinoid to a drug with wide spectrum of action. Rev Bras Psiquiatr 2008; 30: 271-280, doi: 10.1590/S1516-44462008000300015.

15. Bergamaschi MM, Queiroz RH, Zuardi AW, Crippa JA. Safety and side effects of cannabidiol, a Cannabis sativa constituent. Curr Drug Saf 2011; 6: 237-249, doi: 10.2174/ 157488611798280924

16. Malfait AM, Gallily R, Sumariwalla PF, Malik AS, Andreakos $E$, Mechoulam $R$ et al. The nonpsychoactive cannabis constituent cannabidiol is an oral anti-arthritic therapeutic in murine collagen-induced arthritis. Proc Natl Acad Sci USA 2000; 97: 9561-9566, doi: 10.1073/pnas.160105897.

17. Costa B, Colleoni M, Conti S, Parolaro D, Franke C, Trovato $A E$ et al. Oral anti-inflammatory activity of cannabidiol, a nonpsychoactive constituent of cannabis, in acute carrageenaninduced inflammation in the rat paw. Naunyn Schmiedebergs
Arch Pharmacol 2004; 369: 294-299, doi: 10.1007/s00210004-0871-3.

18. Costa B, Trovato AE, Comelli F, Giagnoni G, Colleoni M. The non-psychoactive cannabis constituent cannabidiol is an orally effective therapeutic agent in rat chronic inflammatory and neuropathic pain. Eur J Pharmacol 2007; 556: 75-83, doi: 10.1016/j.ejphar.2006.11.006.

19. Vannucchi MG, Midrio P, Flake AW, Faussone-Pellegrini MS. Neuronal differentiation a myoenteric plexus organization are delayed in gastroschisis: an immunohistochemical study in a rat model. Neurosci Lett 2003; 339: 77-81, doi: 10.1016/S0304-3940(02)01473-8.

20. Pet GE, Stark RA, Meehan JJ, Javid PJ. Outcomes of bedside sutureless umbilical closure without endotracheal intubation for gastroschisis repair in surgical infants. Am J Surg 2017; 213: 958-962, doi: 10.1016/j.amjsurg.2017.03.017.

21. Correia-Pinto J, Tavares ML, Baptista MJ, Estevao-Costa J, Flake AW, Leite-Moreira AF. A new fetal rat model of gastroschisis: development and early characterization. J Pediatr Surg 2001; 36: 213-216, doi: 10.1053/jpsu.2001.20057.

22. Perez M, Benitez SU, Cartarozzi LP, del Bel L, Guimarães FA, Oliveira ALR. Neuroprotection and reduction of glial reaction by cannabidiol treatment after sciatic nerve transection in neonatal rats. Eur J Neurosci 2013; 38: 3424-3434, doi: 10.1111/ejn.12341.

23. Geggel RL, Murphy JD, Langleben D, Crone RK, Vacanti JP, Reid LM. Congenital diaphragmatic hernia: arterial structural changes and persistent pulmonary hypertension after surgical repair. J Pediatr 1985; 107: 457-464, doi: 10.1016/S00223476(85)80534-5.

24. Ding AH, Nathan CF, Stuehr DJ. Release of reactive nitrogen intermediates and reactive oxygen intermediates from mouse peritoneal macrophages. Comparison of activating cytokines and evidence for independent production. J Immunol 1988; 141: 2407-2412.

25. Baird PA, MacDonald EC. An epidemiologic study of congenital malformations of the anterior abdominal wall in more than half a million consecutive live births. Am J Hum Genet 1981; 33: 470 e8.

26. Suzuhigashi M, Kaji T, Nakame K, Mukai M, Yamada W, Onishi $S$ et al. Abdominal wall regenerative medicine for a large defect using tissue engineering: an experimental study. Pediatr Surg Int 2016; 32: 959-965, doi: 10.1007/ s00383-016-3949-3.

27. Gonçalves FL, da Silva R, Schmidt AF, de Oliveira MG, Sbragia L. Hydrogel protection: a novel approach to reduce bowel inflammation in experimental gastroschisis. Eur $J$ Obstet Gynecol Reprod Biol 2010; 148: 35-39, doi: 10.1016/ j.ejogrb.2009.10.009.

28. De Filippis D, Esposito G, Cirillo C, Cipriano M, De Winter BY, Scuderi $\mathrm{C}$ et al. Cannabidiol reduces intestinal inflammation through the control of neuroimmune axis. PLoS One 2011; 6: e28159, doi: 10.1371/journal.pone.0028159.

29. Esposito G, Filippis DD, Cirillo C, luvone T, Capoccia E, Scuderi et al. Cannabidiol in inflammatory bowel diseases: a brief overview. Phytother Res 2013; 27: 633-636, doi: 10.1002/ptr.4781.

30. Franchi-Teixeira AR, Barreto MG, Nogueira B, Bittencourt D, Violin L, Sbragia L. Aminiotic fluid and intrauterine growth restriction in a gastroschisis fetal rat model. Fetal Diagn Ther 2005; 20: 494-497, doi: 10.1159/000088037. 
31. Bueno MP, Gonçalves FL, Guadagnini D, Barini R, Saad MJ, Gallindo RM et al. The role of gut-liver axis in the restriction of intrauterine growth in a model of experimental gastroschisis. Acta Cir Bras 2013; 28 (Suppl 1): 3-7, doi: 10.1590/S0102-86502013001300002.

32. Wasserman E, Tam J, Mechoulam R, Zimmer A, Maor G, Bab I. CB1 cannabinoid receptors mediate endochondral skeletal growth attenuation by $\Delta 9$ tetrahydrocannabinol. Ann N Y Acad Sci 2015; 1335: 110-119, doi: 10.1111/ nyas. 12642.

33. Bealer JF, Graf J, Bruch SW, Adzick NS, Harrison MR. Gastroschisis increases small bowel nitric oxide synthase activity. J Pediatr Surg 1996; 31: 1043-1045 ; discussion 1045-1046, doi: 10.1016/S0022-3468(96)90083-4.

34. Dilsiz A, Gündogan AH, Aktan M, Duman S, Aktung T. Nitric Oxide Synthase Inhibition Prevents Intestinal Damage in Gastroschisis: A Morphological Evaluation in Chick Embryos. J Pediatr Surg 1999; 34: 1248-1252, doi: 10.1016/S00223468(99)90161-6.

35. Esposito G, Scuderi C, Savani C, Steardo L Jr, De Filippis D, Cottone P. Cannabidiol in vivo blunts beta-amyloid induced neuroinflammation by suppressing IL-1beta and iNOS expression. Br J Pharmacol 2007; 151: 1272-1279, doi: $10.1038 /$ sj.bjp.0707337.

36. Bujalska-Zadrożny $\mathrm{M}$, de Cordé $\mathrm{A}$, Pawlik K. Influence of nitric oxide synthase or cyclooxygenase inhibitors on cannabinoids activity in streptozotocin-induced neuropathy. Pharmacol Rep 2015; 67: 209-216, doi: 10.1016/j.pharep. 2014.08.023.

37. Capasso R, Borrelli F, Aviello G, Romano B, Scalisi C, Capasso $\mathrm{F}$ et al. Cannabidiol, extracted from Cannabis sativa, selectively inhibits inflammatory hypermotility in mice. Br J Pharmacol 2008; 154: 1001-1008, doi: 10.1038/ bjp.2008.177.

38. Borrelli F, Aviello G, Romano B, Orlando P, Capasso R, Maiello $\mathrm{F}$ et al. Cannabidiol, a safe and non-psychotropic ingredient of the marijuana plant Cannabis sativa, is protective in a murine model of colitis. $\mathrm{J} \mathrm{Mol} \mathrm{Med} \mathrm{2009;} \mathrm{87:}$ 1111-1121, doi: 10.1007/s00109-009-0512-x.

39. Lin XH, Yuece B, Li YY, Feng YJ, Feng JY, Yu L et al. A novel CB receptor GPR55 and its ligands are involved in regulation of gut movement in rodents. Neurogastroenterol Motil 2011; 23: 862-e342, doi: 10.1111/j.1365-2982.2011. 01742.x. 